\title{
DIDACTOLOGÍA: UNA CIENCIA DE DISEÑO
}

\author{
Anna ESTANY \\ Departament de Filosofia, Universitat Autònoma de Barcelona * \\ Mercè IZQUIERDO AYMERICH \\ Departament Didactica de les Ciències, Universitat Autònoma de Barcelona *
}

Resumen: El objetivo de este trabajo responde a la necesidad de una reflexión teórica sobre la enseñanza de las ciencias, situando la didactologia en el marco de las disciplinas cientificas, definiendo su objeto y estableciendo los términos de la relación intelectual entre la didactología y otras disciplinas.

A partir de la distinción que hace I. Niiniluoto entre investigación básica, investigación aplicada y tecnología, se caracteriza la didactología como un tipo de ciencia aplicada denominada ciencia de diseño en el sentido de una ciencia con un proyecto a llevar a cabo en el curso de su práctica investigadora.

\section{Introducción}

El objetivo de este trabajo responde a la necesidad de una reflexión teórica sobre la enseñanza de las ciencias y tiene como objetivo situar la didactología en el marco de las disciplinas científicas, definir su objeto y establecer los términos de la relación intelectual entre la didactologia y otras disciplinas. En primer lugar, definimos este campo disciplinar como una ciencia de diseño, en el sentido de una ciencia con un proyecto o fin a conseguir. En segundo lugar, examinamos en que consiste el "contrato didáctico" como unidad de transmisión del conocimiento donde confluyen la fundamentación teórica de la didactología y el fin a alcanzar con la enseñanza de las ciencias. Finalmente, abor-

* Este artículo ha sido elaborado dentro del Proyecto de Investigación PB-98-0495-C0801 sobre "Hacia una metateoría interdesciplinar de la ciencia" coordinado al Proyecto "Ciencia y valores" financiado por el Ministerio de Educación y Cultura. 
damos los modelos didácticos como primer paso para llegar a una teoría que englobe dichos modelos.

La educación y los procesos a ella ligados son uno de los fenómenos más importantes de una sociedad y un buen termómetro de los valores prevalecientes en la misma. Incluso en el caso de que algunos países o algunos gobernantes no la consideren una cuestión prioritaria, el desinterés o minusvaloración es también, por eso mismo, revelador de la naturaleza de esta sociedad y de sus gobernantes. La educación como fenómeno social junto a los procedimientos y medios de impartirla y llevarla a efecto ha sido y es objeto de estudio por parte de la comunidad académica y, en las últimas décadas, a partir de las didácticas específicas, ha adquirido un desarrollo muy importante construyéndose un nicho disciplinario.

La educación como disciplina ha tenido varias denominaciones, dentro y fuera de nuestro país: "pedagogía", "ciencias de la educación", "didáctica", entre las más habituales. Debido a una serie de circunstancias históricas algunas de las denominaciones están cargadas de connotaciones peyorativas. El estado actual de esta disciplina, concretada en la enseñanza de las ciencias, muestra que en su seno existe una auténtico debate intelectual entroncado con los debates en la ciencia y en la filosofía. Esto nos ha llevado a proponer una nueva denominación: "didactologia», término que responde mejor a los intereses y objetivos de esta disciplina ${ }^{1}$.

En el momento histórico en que vivimos "enseñar ciencias" es algo muy complejo. Hasta ahora se pensaba que la didáctica era una metodología para enseñar bien los programas de ciencias establecidos. Pero en la actualidad se han hecho evidentes varios problemas que han transformado la enseñanza de las ciencias en algo muy diferente. (Gutiérrez, 1985) (Azcárate, Espinet, Izquierdo, Sanmartí, 1993).

En primer lugar, los alumnos llegan a la escuela con sus propias ideas sobre el funcionamiento del mundo, y estas ideas o "miniteorías" son muy difíciles de cambiar (Driver, 1986); por tanto, para enseñar eficazmente las ciencias es necesario tener en cuenta estas ideas (Black y Lucas, 1993) (Ogborn, 1985). En segundo lugar, el espectacular desarrollo de los conocimientos científicos a enseñar y la inclusión de las ciencias en la escuela obligatoria, que afecta a toda la población hasta los 16 años, obliga a seleccionar los conocimientos que se consideran más

1 Lo que aquí denominamos "didactología" corresponde a lo que en la literatura más reciente se ha denominado "didáctica de las ciencias". 
importantes (Fensham, 1988). Finalmente, las implicaciones y consecuencias sociales, ambientales y éticas de las ciencias requieren un estudio interdisciplinar que no se había previsto anteriormente (Izquierdo, 1994b). Los conocimientos a enseñar pueden seleccionarse de muchas maneras diferentes, pueden referirse a muchos campos distintos y llegan a configurar objetivos de enseñanza dispares. La organización del curriculum pone ahora en manos del profesor de ciencias la responsabilidad de construir sus propias "ciencias escolares", a diferencia de lo que ocurría hace poco, cuando parecía que existían unos contenidos básicos indiscutibles. Esto nos lleva a tener en cuenta ahora las relaciones entre ciencia, técnica y sociedad, la epistemología e historia de las ciencias, la educación ambiental, la educación para la salud, el conocimiento de los nuevos materiales, etc.

Por todo ello "enseñar ciencias», a pesar de ser una profesión muy antigua, plantea ahora nuevos problemas, que pueden resolverse mediante las aportaciones teóricas de campos disciplinares como las ciencias cognitivas, la sociología y las ciencias de la información, que enriquecen las tradicionales, surgidas de la práctica en el aula y que son más propiamente pedagógicas. (Yager et al., 1982). Aparece así la didactología o "la ciencia de enseñar ciencias", que puede ser considerada ahora una nueva disciplina emergente, un nuevo campo científico que tiene que ver con la planificación, la ejecución y la evaluación de esta enseñanza en base a un planteamiento teórico. Los cambios en las propias ciencias y su espectacular desarrollo, los nuevos campos disciplinares que aparecen, la nueva historia y filosofla de las ciencias, junto con lo que ahora se sabe de los mecanismos psicológicos, sociales y lingüísticos que dan sentido al mundo, hacen tan complejo el proceso de determinar cuáles son los componentes de la enseñanza de las ciencias que cada vez es más evidente que saber alguna de las ciencias y saber cómo enseñarlas a un grupo concreto de personas son dos cosas diferentes y requiere conocimientos diferentes. (Aliberas, Gutiérrez, Izquierdo, 1990).

\section{La Didactología, en el marco de las disciplinas científicas}

Si nos preguntamos si la didactología es una ciencia, veremos que es imposible dar una respuesta categórica. Cuando pensamos en ciencias lo primero que se nos ocurre es la física, la biología o la sociología. Suponiendo que contestáramos que la didactología es una ciencia, posiblemente diríamos que no es como las citadas anteriormente. Para esta cuestión son esclarecedoras las distinciones que hace I. Niiniliuoto (1993) sobre los diversos tipos de investigación. 
Niiniluoto señala que la mayoría del trabajo realizado por los filósofos de la ciencia tiene como punto de mira las ciencias básicas como la matemática, la física, la biología, la psicología y la sociología, pero que se ha prestado mucha menos atención, por no decir nula, a campos como matemática aplicada, veterinaria, logopedia, dietética, farmacología, ingeniería etc., a pesar del peso que dichos campos disciplinarios tienen en el conjunto de la investigación y en las instituciones académicas, sin contar la trascendencia para el conjunto de la sociedad, al menos en el mundo occidental. A lo largo de la historia de la filosofía de la ciencia se ha caído en varios errores como consecuencia de la falta de distinción entre ciencia básica y ciencia aplicada. Por un lado, se han tomado todas las ciencias como básicas. Esto es lo que ha sucedido hasta ahora aunque este supuesto no estuviera explícito claramente. Así, por filosofia de la ciencia se ha entendido filosofia de las ciencias fundamentales. Por otro lado, desde un punto de vista opuesto, se han tomado todas las ciencias como aplicadas y de esta manera, las reflexiones filosóficas sobre ciencia aplicada se han extrapolado a todas las ciencias sin distinción de básicas o no. Tal es el caso del filósofo alemán J. Habermas (1971) que al hablar de las ciencias como una actividad humana con «interés técnico" para controlar al mundo está atribuyendo a las ciencias básicas lo que es sólo atribuible a las ciencias aplicadas.

La concepción de Niiniluoto reside en situar la ciencia aplicada entre las ciencias básicas y la tecnología. Las ciencias básicas definen sus objetivos a partir de valores puramente epistémicos, básicamente verdad, información y poder explicativo. En cambio la tecnología define sus objetivos en función de la eficiencia de los artefactos materiales y sociales para la transformación de la realidad. En este sentido es importante señalar que la diferencia crucial entre aceptar una hipótesis científica y construir un artefacto es que mientras en el primer caso la hipótesis tiene un valor de verdad con o sin consecuencias, independiente de los efectos de la investigación de la realidad social u otros que promueva, en el segundo, el objetivo de la construcción de un artefacto es precisamente el de ser un instrumento para la modificación de la realidad social u otras realidades. El que el agua sea $\mathrm{H}_{2} \mathrm{O}$ tiene un valor de verdad independientemente de si esto perjudica o no a nuestra especie, pero la construcción de una planta nuclear o el uso de fertilizantes tiene unas consecuencias directas sobre la producción de energía eléctrica y de alimentos, y sobre la flora y fauna. De las consecuencias que se deriven no podemos prescindir a la hora de tomar la decisión de construir el artefacto con finalidad eficiente. 
La ciencia aplicada estaría entre la ciencia y la tecnología: por un lado, produce conocimiento; por otro, se intenta que este conocimiento repercuta en algún tipo de actividad humana y social e incremente, así, la efectividad del fin propuesto. Es decir, las ciencias aplicadas se valoran, simultáneamente, por valores epistémicos y prácticos. Entre las ciencias aplicadas son especialmente pertinentes aquellas que Niiniluoto califica de "ciencias de diseño" («design science») como la ingeniería, la medicina, la economía, la pedagogía, que tienen que ver con un proyecto, es decir, no pretenden decirnos cómo "son" las cosas sino cómo «tendrían que ser» para alcanzar determinados fines y ejercer determinadas funciones. Interesa cómo son las cosas pero sólo en la medida que la información sea necesaria para alcanzar el fin deseado. El conjunto de información pertinente para alcanzar el fin propuesto por una ciencia de diseńo es lo que podríamos llamar el "pilar teórico" sobre el que se asienta dicha ciencia de diseño.

La didactología sería una ciencia de diseño en la medida en que teoriza sobre la transmisión y construcción del conocimiento y sobre los procedimientos que las hacen posibles. La didactología tendría varias ramas en función del tipo de conocimiento que queramos transmitir y que constituyen las diversas didácticas especiales, por ejemplo, didáctica de la física, de la matemática, de la historia, etc. Son las didácticas especiales las que estudian los mejores procedimientos y técnicas adecuadas para llevar a cabo la enseñanza, pero todas ellas comparten las mismas reglas de juego y los mismos principios teóricos atribuidos a la didactología.

Probablemente no hay diferencias importantes entre las didácticas especificas, al menos a nivel de los principios teóricos que la fundamentan. Es decir, lo que digamos sobre la transmisión y construcción del conocimiento cientifico en general es atribuible a un conocimiento científico particular, sea éste de física, de biología o de geografía. Desde el punto de vista de la transmisión de conocimiento no hay distinción radical, es decir, no se rigen por principios teóricos radicalmente distintos las ciencias humanas, naturales y sociales. Sin embargo, podemos encontrar diferencias en el nivel que llamamos "didáctico" que responden a las diferencias entre las disciplinas cientificas. La relación entre la didactología y las didácticas particulares tiene que ser de mutua interacción, es decir, ni una didactologia «a priori» desvinculada de las didácticas particulares ni unas didácticas particulares sin fundamento teórico.

A partir de esta caracterización de ciencia de diseño podemos decir que el objetivo de la didactología es la transmisión de conocimientos y de valores a 
los individuos de una sociedad en el marco de una cultura determinada, del mismo modo que la medicina tiene como objetivo prevenir y curar las enfermedades y la ingeniería perfeccionar la técnica industrial. Todas las ciencias de diseño tienen una vertiente que tiene que ver con los conocimientos científicos que utiliza para llevar a cabo el propósito, son los pilares de su fundamentación teórica; la forma de valorar esta vertiente es puramente epistémica. Pero estas ciencias tienen otra vertiente que tiene que ver con el objetivo propuesto, su valoración es práctica y tiene que ver con el campo implicado.

\section{El contrato didáctico}

El contrato didáctico constituye una forma de canalizar las dos vertientes implicadas en la didactología: la fundamentación teórica y el fin a alcanzar. Joshua y Dupin (1993) señalan que en la transmisión del conocimiento intervienen tres elementos: el profesor, el alumno y el saber.

\section{Profesor \\ (con su ideología privada) \\ contrato didáctico}

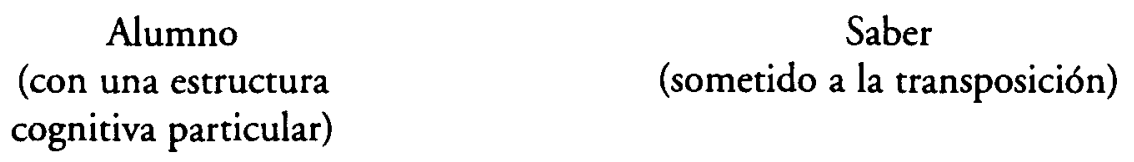

El objetivo de la enseñanza de las ciencias es comprender cómo funciona la interacción entre los tres elementos y cómo tiene que ser el "contrato didáctico" para que la enseñanza sea operativa (Brousseau, 1984). De aquí surgen problemas que pueden concretarse a partir de una serie de preguntas que se nos han hecho ya familiares: ¿qué enseñar? ¿cuándo enseñar? ¿cómo enseñar? ¿cómo evaluar los resultados? Estas preguntas y muchas más nos llevan a la búsqueda de la teoría explicativa, para la cual tenemos que recurrir a campos de conocimiento muy diversos y a marcos teóricos variados y complementarios como los siguientes: las propias materias científicas cuya enseñanza se plantea y que tienen una problemática y una estructura específica; la historia y la epistemología de estas materias científicas, que explican su génesis y su desarrollo; la sociología, que permite plantearse la interdependencia entre ciencia y sociedad y su influencia en la formación de los individuos de una sociedad democrática cada vez más inmersa en la tecnología; la lingüística, que es 
fundamental para comprender muchas de las causas de los aprendizajes difíciles; la psicología, que aporta el conocimiento del desarrollo de la personalidad y de los modelos cognitivos para las actividades y los procesos de aprendizaje; la pedagogía, que aporta el análisis de las relaciones entre enseñanza y aprendizaje en el marco de las instituciones escolares; la propia práctica profesional; otras áreas, como la comunicación, la tecnología, la inteligencia artificial, etc. Los problemas resueltos van configurando un "marco teórico y metodológico" que ya es propio de la didactología y que no se puede considerar como una simple suma de las áreas de conocimiento que las fundamentan.

\section{Fundamentación teórica de la enseñanza de las ciencias}

Para fundamentar teóricamente la enseñanza de las ciencias hay que examinar los pilares teóricos, las disciplinas afines y los elementos que entran en acción en el contrato didáctico.

Uno de los pilares teóricos fundamentales es la psicología cognitiva y tiene que ver con el profesor, el alumno y la relación profesor/alumno; otro de los pilares es la filosofía de la ciencia y tiene que ver con la relación profesor/saber y alumno/saber, y esta relación es mediada por el contrato didáctico, puesto que el alumno no tiene acceso directo al saber más que a través del profesor, al menos del saber sistematizado en el marco de la institución escolar.

Además de los pilares fundamentales de esta trilogía, en esta relación está el producto a transmitir, en este caso, la ciencia. La ciencia se ha de tomar tanto en el contexto de la justificación, estado de la cuestión a que ha llegado el saber en el momento en que se realiza la transmisión y su evaluación epistémica, como en el contexto del descubrimiento, proceso por el que se ha llegado a dicha situación, es decir, la historia de la ciencia.

Por lo tanto, podemos afirmar que hay tres materias en que el enseñante de ciencias tiene que ser competente para poder llevar con éxito su labor: psicología cognitiva, filosoffa de la ciencia y la ciencia transmitida, además de la historia de la misma. Todos estos conocimientos son absolutamente necesarios para la formación del profesorado de cualquier disciplina. Para completar este cuadro hay que incluir lo propiamente didáctico como las técnicas concretas de la transposición didáctica en las que los didactólogos son expertos (Benedito, 1987) (Joshua y Dupin, 1993) (Jorba y Sanmartí, en prensa). 
Existen también unos campos afines que tienen que ver con el propósito de la didactología como ciencia de diseño. En el objetivo de la misma está implicada toda la sociedad que incide en dichos objetivos. Estos dependerán, por ejemplo, de la política educativa. Es aquí donde entran en acción los factores sociales y políticos y, por tanto, ideológicos. En este sentido tiene mucho que decir la sociología de la educación y las políticas educativas así como los valores y orientaciones educativas de una sociedad determinada.

\subsection{La psicología cognitiva}

La psicología cognitiva constituye uno de los pilares teóricos de la didactologia y tiene que ver con la relación profesor-alumno. De lo que se trata dentro del contrato didáctico es de conseguir que el alumno domine una serie de conocimientos. Para que esta operación tenga éxito el alumno tiene que ser capaz de procesar la información, almacenarla y poder utilizarla cuando tenga necesidad de ella, lo cual significa ser capaz de recuperar dicha información en el momento oportuno. Todas estas operaciones forman parte de la actividad cognitiva, objeto de estudio de la psicología cognitiva. Esta disciplina forma parte del campo interdisciplinar denominado "ciencia cognitiva" (Andersson, 1977) (Schank y Abelson, 1977) (Stewart, 1985) (Minsky, 1988) (Pozo, 1989).

El campo de investigación de la ciencia cognitiva, a pesar de ser relativamente joven, ha sufrido cambios importantes desde su nacimiento, hasta el punto de que se habla a veces de dos paradigmas en el seno de la psicología cognitiva. Ambos están interesados por los fenómenos mentales, por modelar la actividad cognitiva y por los sistemas computacionales, características que les diferencia de la psicologia conductista que gira en torno de la conducta animal y humana. Además, para la psicología cognitiva, de uno u otro signo, la conducta sólo es pertinente en tanto en cuanto refleja los estados mentales. Sin embargo, existen diferencias significativas entre el «enfoque simbólico o de procesamiento de la información" y el "enfoque conexionista o de procesamiento de distribución paralelan de la psicología cognitiva.

Para el enfoque simbólico aprender un lenguaje consiste en aprender un conjunto de reglas contenidas en algo parecido a un programa informático en vista a una manipulación de símbolos. La metáfora que utiliza este enfoque para entender el funcionamiento de la mente es el ordenador. Por ejemplo, en una calculadora de bolsillo los diversos estados de entrada representan números especificos y 
operaciones aritméticas y lo que ocurre a continuación en el interior está determinado por las características computacionales relativas a dichos estados. Los estados de salida se relacionan mediante reglas con los respectivos estados de entrada. Tomando el ordenador como modelo y extrapolándolo a los organismos inteligentes vemos que la única diferencia es que sus estados de entrada representan muchas más cosas que números y las operaciones aritméticas corresponden a funciones cognitivas que pueden llegar a ser muy complejas. La idea es construir y configurar una máquina física que actúe de forma inteligente lo cual quiere decir que si esta actuación fuera de un humano sería considerada inteligente. El área de conocimiento que tiene como objetivo diseñar estas máquinas es la denominada «inteligencia artificial» (LA) (Johnson-Laird, 1988).

El enfoque conexionista propone sustituir la metáfora del ordenador por la metáfora del cerebro. Es decir, en lugar de reconstruir los fenómenos cognitivos a partir de la forma en que los ordenadores procesan la información, el conexionismo toma la forma de procesar la información que tiene el cerebro como modelo de la arquitectura que deberían tener las máquinas informáticas. Esto significa que, a pesar de que ambos enfoques tienen un pie en la ciencia de la computación y otro en la neurobiología, el enfoque simbólico parte de la primera y el conexionista parte de la segunda. A partir del modelo biológico como guía heurística en la investigación en la ciencia de la computación la labor en lo que podríamos denominar la "segunda etapa de la inteligencia artificial" consiste en crear un tipo de arquitectura de los sistemas inteligentes, que simulan la forma de cognición humana, denominados "redes neuronales" y que son entidades puramente formales y artificiales (Rumelhardt y Norman, 1978).

Desde la perspectiva de la ciencia cognitiva la didactología tiene que tener en cuenta los logros tanto en neurología como en ciencia de la computación. Estas ciencias proporcionan el conocimiento indispensable, aunque no suficiente, para llevar a cabo con éxito la transmisión de conocimiento, ya que estas ciencias nos dicen cómo percibimos, cómo mantenemos la atención, cómo recordamos y cómo ejercemos nuestra capacidad creativa. Una de las claves de la fundamentación teórica de la didactología está en encontrar las bases neurobiológicas de nuestra actividad cognitiva.

\subsection{Las aportaciones de la filosofia de la ciencia}

Otra de las relaciones en el contrato didáctico es la de alumno-saber, relación mediada por el profesor. El profesor "coge" el saber y lo "pasa» al alumno, pero 
¿qué es lo que le transmite?, ¿cuáles son las categorías que el profesor utiliza para organizar el saber y transmitirlo? El profesor no le transmite el saber «en bruto" sino "reconstruido". La filosofía de la ciencia interviene cuando nos planteamos las guías de esta reconstrucción (Hodson, 1985) (Izquierdo, 1990, 1994 a).

La filosofía de la ciencia es una reflexión metateórica de la ciencia. En un sentido general podemos decir que nació con la propia ciencia, así vemos que Aristóteles no solamente escribió sobre física y sobre astronomía sino también sobre la estructura lógica del razonamiento. Sin embargo, como una rama independiente de la filosofía es un fenómeno mucho más reciente que podemos situarlo en la constitución del Círculo de Viena en el primer tercio de nuestro siglo. Esta reflexión filosófica puede hacerse desde tres aspectos distintos: i) el metodológico, ii) el ontológico, y iii) el lógico-semántico (Estany, 1993). El aspecto metodológico es muy importante en el conjunto de la filosofía de la ciencia hasta el punto de que a veces la filosofía de la ciencia se denomina "metodología de la ciencia». La metodología proporciona las guías y principios que deben regir en la investigación científica. Por ejemplo, la metodología analiza los tipos de conceptos científicos, las características de una definición, la distinción entre términos teóricos y observacionales, los modelos de explicación científica, las características de las leyes de la naturaleza y la estructura de las teorías científicas. Como cuestiones afines al aspecto metodológico pero que no constituyen el meollo de lo que se entiende por metodología de la ciencia están, por un lado, las técnicas de investigación, muy específicas de cada disciplina, y por otro, los principios metafísicos que están en el sustrato de la investigación científica.

El aspecto ontológico discurre en varios niveles: uno consiste en determinar las unidades mínimas sobre las que se construyen las leyes y las teorías, por ejemplo, los átomos para la teoría atómica, los elementos químicos para la química de Lavoisier, o las partículas elementales para la física de altas energías; el otro nivel consiste en determinar cuáles son las categorías fundamentales de la ciencia, por ejemplo, hay concepciones que consideran las teorías como la única forma de presentar la ciencia, pero otras concepciones consideran que los resultados de la ciencia pueden exponerse en forma de modelos, de tipologías etc.

Por último, el aspecto lógico-semántico proporciona la estructura lógica del discurso científico, siendo uno de sus objetivos la reconstrucción formal de teorías científicas. $\mathrm{El}$ aspecto formal de la filosofía de la ciencia fue pre- 
dominante en la primera etapa de institucionalización de la filosofía de la ciencia hasta el punto de que se la entendía como "lógica de la ciencia».

La ciencia expresa sus resultados con un aparato conceptual que va desde categorías simples como los conceptos hasta categorías muy complejas como las teorías. Analizar las reglas de juego de la ciencia significa analizar este aparato conceptual que, en sentido propio, deberíamos llamar "metaconceptual».

El profesor transmite el saber en forma de conceptos, leyes y teorías científicas. Todo este saber tiene un objetivo último que es explicar el mundo, pero esto implica que hemos reflexionado sobre qué es una explicación científica, tema central en filosofía de la ciencia. Podemos encontrar varias concepciones sobre la explicación científica, sobre la estructura lógica de las categorías con las que reconstruimos el saber pero es indudable que la transmisión del conocimiento científico requiere un análisis previo de las cuestiones metateóricas.

\subsubsection{Modelos cognitivos de ciencia}

De especial relevancia para la didactología son los modelos cognitivos de ciencia, surgidos por el impacto de las ciencias cognitivas en la filosofía. La emergencia de la ciencia cognitiva, lugar de encuentro de disciplinas como la inteligencia artificial, la psicología cognitiva, la neurociencia, la lingüística y la epistemología, ha hecho que algunos filósofos de la ciencia, interesados en conocer el origen y formación del conocimiento humano, propugnen un nuevo enfoque, según el cual la ciencia es una actividad cognitiva y la filosofía de la ciencia utiliza conceptos y métodos de las ciencias cognitivas para el estudio de dicha actividad. Desde esta perspectiva las ciencias aparecen como empresas profundamente humanas, y su meta es interpretar el mundo, utilizando para ello la "racionalidad moderada" (la capacidad humana de emitir juicios y de progresar hacia una meta). El enfoque cognitivo en filosofía de la ciencia ha hecho que en el análisis metateórico de la ciencia se tenga en cuenta no sólo la ciencia como producto sino también el proceso cognitivo mediante el cual los científicos llegan a este producto.

Uno de los filósofos que ha trabajado con orientación cognitiva es R. Giere (1988), quien destaca la importancia de los «modelos teóricos» en la representación del conocimiento científico, considerados un tipo de representación mental semejantes a "mapas internos" del mundo externo. La relaciones entre 
los modelos teóricos y la realidad son de similitud, no de correspondencia, y se establecen mediante "hipótesis teóricas". Los modelos no son ni verdaderos ni falsos sino más o menos similares a los sistemas reales; pero las hipótesis teóricas hacen afirmaciones sobre los fenómenos teniendo los modelos como guía, y por tanto son verdaderas o falsas, y tienen contenido empírico. Una teoría científica sería, así, un conjunto de modelos más un conjunto de hipótesis teóricas.

Puede comprenderse fácilmente la importancia que tienen los modelos cognitivos de ciencia para la fundamentación de la intervención docente en ciencias, puesto que en ellos están contemplados a la vez los aspectos epistemológicos y psicológicos. Creemos que aún no han sido exploradas las consecuencias de esta nueva situación para la didáctica de las ciencias. Consideraremos que los llamados «modelos didácticos" son modelos teóricos en el sentido de Giere y que, por tanto, constituyen una aportación muy importante para la construcción progresiva de la teoría didactológica.

\section{Los fines de la didactología y su vinculación al elemento social del contrato didáctico}

Los fines u objetivos de la didactología constituyen la vertiente por la que se considera una ciencia de diseńo y es a través de los fines que todo el contrato social entra en juego. Los estudios en el campo de la didactología han prestado especial atención a los aspectos sociales del fenómeno educativo, influenciados, implícita o explícitamente, por corrientes sociologistas, lo cual ha provocado una cierta confusión conceptual que ha revertido desfavorablemente en este campo disciplinario.

La primera confusión es entre el concepto de educación en general y el de educación científica. La didactología versa sobre la educación científica y no sobre la educación en general. La educación es un concepto mucho más amplio que incluye la educación científica entendida como la transmisión de conocimiento. En la educación (las instituciones que tiene que hacerse cargo de ella, familia, escuela etc.) deberán incluirse temáticas y fenómenos que no forman parte de la transmisión de conocimiento en sentido estricto, tales como la formación de hábitos, las interiorización de normas éticas, el comportamiento cívico, el desarrollo de habilidades artísticas (música, pintura, etc.), la coordinación motora del propio cuerpo (rítmica, deporte, etc.) que, 
siendo tan importantes para la educación del individuo como la adquisición del conocimiento deben ser tenidos en cuenta al determinar los objetivos de la didactología pero no identificarse con ellos.

Lo dicho hasta ahora no significa que la didactología no esté vinculada a la sociedad pero es necesario distinguir y determinar exactamente dónde reside la incidencia de lo social para no sucumbir en un mar de confusiones. Su relación con los factores sociales procede de las especiales características de las ciencias de diseño, debido a que sus enunciados tienen una estructura lógica distinta de los enunciados con que la investigación básica expresa sus resultados (Niiniluoto, 1993). La estructura lógica de un enunciado de investigación básica tiene una de las formas siguientes según estemos en un sistema determinista o probabilista:

(1) X causa A en situación B.

(2) $\mathrm{X}$ tiende a causar $\mathrm{A}$ (con probabilidad $\mathrm{p}$ ) en situación $\mathrm{B}$.

En cambio la estructura lógica de los enunciados de una ciencia de diseño tiene la estructura siguiente:

(3) Si quieres A y crees que estás en la situación $\mathrm{B}$, tienes (o deberías, o es racional) que hagas $\mathrm{X}$.

El problema que se nos plantea es que los enunciados normativos carecen de valor de verdad lo cual nos plantea la cuestión de cómo pueden constituir conocimiento. $\mathrm{G}, \mathrm{H}$, von Wright (1979 y 1983) nos proporciona una solución con su noción de "norma técnica» que es un enunciado factual sobre la relación entre medios y fines.

Por ejemplo, tomemos la norma siguiente:

(4) Si quieres hacer la cabaña habitable, tienes que calentarla.

Tanto el antecedente como el consecuente de esta norma condicional son enunciados descriptivos, el primero sobre los deseos y preferencias de una persona, el segundo sobre la existencia de un "deber técnico" para ella. La norma técnica (4) es verdadera si y solo si calentar la cabaña es una condición necesaria para hacerla habitable.

Por lo tanto podemos decir que la verdad o falsedad de (3) es una característica objetiva del mundo que no presupone compromiso alguno con la valoración de su antecedente. A partir de esta estructura de los enunciados de las 
ciencias de diseño podemos señalar la incidencia de lo social. El elemento social está en la fijación de "A" que es un fin a alcanzar, y el objetivo que persigue una ciencia de diseño determinada está en la disponibilidad de los recursos para alcanzar "X" y está en la definición de "B" que es una realidad social. Los debates políticos son especialmente pertinentes para fijar " $A$ », definir «B» y alcanzar "X".

En el caso de la didactología las distintas prioridades sociales y políticas estarán presentes a la hora de confeccionar los curricula de las diversas disciplinas científicas, pero no porque el saber a transmitir esté cargado de ideología. La ciencia fundamental describe el mundo, nos dice cómo es el mundo no cómo debería ser el mundo, esto no depende de nosotros, y esto vale para todas las ciencias, formales y empíricas. En lo que sí intervienen los valores es cuando se prima un conocimiento sobre otro a la hora de confeccionar un curriculum.

La diferencia entre la didactología y otras ciencias de diseño como la medicina o la economía consiste en que las últimas han llegado a un consenso social respecto a los fines. Así la medicina aspira a lograr la salud de los individuos y la economía a maximizar las ganancias, en cambio la didactología no tiene claros los fines ya que no hay consenso social respecto a los objetivos. Sin embargo, hay que señalar que incluso en ciencias tan consensuadas como la medicina y la economía cabe la discusión de los fines ya que a veces si bien hay consenso, no hay unanimidad sobre el concepto de salud o sobre si maximizar los beneficios monetarios tiene que primar por encima de cualquier otra variable social.

Volviendo a los fines de la didactología, "A" puede consistir en lograr un mínimo de educación científica para toda la población o puede consistir en dar a la población distintos niveles de conocimiento. En este caso puede ser que los criterios sean según el nivel o las habilidades individuales o según el nivel económico. Los curricula serán distintos según los valores que prevalezcan en una sociedad determinada. Estos fines pueden ir de más general a más concreto. Para nuestra sociedad occidental los valores últimos siguen siendo los de la revolución francesa: igualdad, libertad y fraternidad. Según cuál de ellos se prime tendremos un tipo de sociedad u otra y a partir de aquí se irán concretando los valores más próximos. En los países del llamado "socialismo real" se primaba la igualdad en aras de la libertad, haciendo caso omiso de la fraternidad. Las politicas neoliberales "a lo Tatcher-Reagan-Pinochet" defendidas por economistas como Friedman priman la libertad en aras de la igualdad y de la fraternidad. 
Entre estos dos extremos se encuentran regímenes políticos intermedios que intentan mantener un cierto equilibrio entre estas tres coordenadas.

Por tanto, el debate político y los factores sociales son perfectamente identificables y están claramente situados. No están ni en la investigación básica ni en los pilares teóricos en los que se asienta la didactología. La incidencia social está en la fijación de los fines propuestos en la educación científica y no cabe duda de que la escala de valores de una sociedad determinada revertirá sobre los fines a alcanzar en la educación científica, es decir, en la transmisión de conocimiento a los individuos (Krugly-Smolska, 1995) (Hodson, 1985).

\section{Los Modelos Didácticos}

La investigación específica en la enseñanza de las ciencias avanzará en la medida en que seamos capaces de coordinar las teorías procedentes de las diversas disciplinas pertinentes para la didacticología y de adaptarlas de manera pragmática a la realidad que se explora. Esta realidad es el aula y los problemas que allí se plantean. A partir de dichos problemas se configurarán finalmente los modelos teóricos propios de la didactología que permiten resolver los problemas. Todo lo dicho hasta ahora tiene que quedar reflejado en dichos modelos.

Actuando de esta guisa podemos encontrarnos con diversidad de «modelos teóricos didácticos" (MTD). Todos ellos son teorías parciales porque sólo se aplican a los problemas que han sido resueltos con ellos. Sin embargo, todo ello es propio de una disciplina emergente y es más bien beneficioso, porque se ha de intentar resolver los problemas didácticos, aunque sea con una diversidad de modelos. Más adelante, la propia disciplina unificará algunos de los modelos dando lugar a teorías (con lo cual se unificarán también los significados de sus respectivos lenguajes teóricos) y rechazará, quizás, a otros (López Rupérez, 1990) ${ }^{2}$.

Vamos a analizar el modelo de Cañal y Porlán (Cañal, 1987) como ejemplo de modelo didáctico. Según Cañal y Porlán un modelo didáctico debe contener

2 Debemos hacer notar que en esta primera aproximación consideramos sinónimos los términos "modelo didáctico" y "modelo currricular" y, por ello, aceptamos que la teoría didáctica futura será, como ha hemos insinuado, una teoría curricular. Procediendo paso a paso, podríamos imaginar una teoría parcial que unificara los diferentes modelos. 
como mínimo respuestas explícitas o implícitas para las siguientes cuestiones fundamentales: el modelo conceptual con el cual se va a describir el aula, los fines educativos seleccionados y los principios didácticos fundamentales que se ofrecen. De acuerdo también con Coll (1986), proponemos que un modelo didáctico (es decir, un modelo teórico de la didactología) quede caracterizado por su fundamentación (psicológica, epistemológica, sociopedagógica) y principios didácticos resultantes; por sus elementos (finalidad, contenidos, comunicación, organización: recursos, actividades, secuenciación) y por la relación entre los elementos.

El modelo didáctico de Cañal y Porlán, «Modelo basado en la Investigación» (Cañal, 1987) corresponde a un enfoque constructivista ${ }^{3}$ que se distingue por las ideas previas de los alumnos y por el cambio conceptual inspirado en las características de las cambios en las teorías científicas a lo largo de la historia. En líneas generales, podemos decir que un Modelo Teórico Didáctico de corte constructivista se fundamenta en los siguientes "principios didácticos":

1. Un enfoque ambiental, por considerar que puede ayudar a superar el aislamiento del centro escolar respecto al entorno y facilitar una aproximación investigadora al mismo. La investigación directa del entorno aparece como el vínculo entre todos los aprendizajes realizados, tanto en la escuela como fuera de ella.

2. La predisciplinariedad y la interdisciplinariedad, como contrapuestas a una aproximación según la lógica interna de la disciplina y más de acuerdo a las características del estudio de la realidad sociocultural, cuya elaboración se deja a la elaboración personal, fruto de la estructuración de las distintas adquisiciones puntuales que ha realizado el alumno.

3. La comunicación (escuela-medio, profesor-alumno, profesor-profesor, entre alumnos..), como uno de los factores clave en la metodología, tanto la científica como la escolar. Esta última es uno de los pilares de este modelo.

4. La libertad y la cooperación, como factores necesarios para que puedan realizarse los tres anteriores y que determinan un nuevo estilo de relaciones no autoritarias en el aula.

3 El término "constructivista» aplicado a los modelos didácticos no tiene nada que ver con su uso en sociología del conocimiento (muy especialmente en el llamado "Strog Programme in Sociology of Knowledge" donde "constructivismo" se usa en el sentido de que la ciencia es una construcción social. 
Este modelo didáctico aborda una serie de cuestiones que pueden considerarse como "elementos del modelo»:

a. Objetivos: son "metas a comprobar" y no se constituyen en metas obligatorias para todos los alumnos. Se formulan en términos generales, que pueden deglosarse en otros más adecuados para orientar las tareas de enseñanza. Los "puntos de anclaje» (Host, 1982), los "conceptos estructurantes" (Giordan, 1983) y los "conceptos inclusores" (Novak, 1982) serían objetivos conceptuales de este modelo.

b. Contenidos: se definen como "el conjunto de toda la información que se pone en juego en un proceso de enseríanza aprendizaje». No constituyen «lo que hay que aprender" sino que tienen un carácter instrumental y son un medio para nuevos aprendizajes: algunos que son previsibles y figuran, por lo tanto, como "objetivos" y otros que no lo son.

c. Relaciones de comunicación:: las dificultades que existen para que ésta pueda producirse de manera efectiva a fin de poder desarrollar las estrategias adecuadas.

d. Organización: deberá ser flexible y funcional, tanto en horarios como en agrupamientos de alumnos y en la realización de las actividades, que a menudo deberán ser simultáneas y en distintos espacios.

e. Medios técnicos:: se contempla la posibilidad de prescindir del libro de texto y formar una biblioteca de clase

El conjunto de principios y elementos constituyen el modelo teórico en el sentido de Giere, a partir del cual surgirían las hipótesis teóricas que nos darían ideas de hasta qué punto el modelo se asemeja al sistema real (en este caso, el aula).

En el modelo de Porlán y Cañal el "Sistema Aula» adquiere la máxima importancia. En otros modelos no es así. A medida que seamos capaces de generar modelos didácticos bien definidos iremos analizando las ventajas e inconvenientes de cada enfoque y seremos capaces de generar "modelos de los modelos" cada vez más capaces de captar la diversidad de situaciones que se dan en el aula y las posibilidades educativas de cada una de ellas.

\section{Conclusiones}

La didactología es la ciencia que estudia los fenómenos de enseñanza de las disciplinas científicas, las condiciones de transmisión de la cultura científica y 
las condiciones de adquisición de conocimiento por parte del que aprende. También le corresponde decidir qué tipo de ciencia enseñar y cuáles son los componentes de la educación científica. Para cumplir estos objetivos la didactología, además de sustentarse en los modelos teóricos propiamente didácticos, tiene que hacerlo también en la psicología cognitiva, en la filosofía de la ciencia y en la historia de la ciencia y, como campos afines, en la sociología. Sin embargo no hay que olvidar nunca que para enseñar ciencia es condición necesaria, pero no suficiente, conocer a fondo lo que se quiere enseñar. Por lo tanto, no es posible enseñar física o sociología si antes no se tiene un conocimiento de estas ciencias. Sólo después de estar en posesión de los contenidos podemos reflexionar sobre cómo transmitir este conocimiento y sobre cómo crear las condiciones que permitan su adquisición por parte del que aprende.

Ahora empieza a ser posible describir lo que pasa cuando se enseñan ciencias (cómo funciona la interacción alumno-profesor-materia a enseñar, en un espacio social determinado) y empiezan a formularse teorías al respecto. La complejidad de los problemas que deben resolverse requiere un estilo de investigación que sea capaz de captar todos los detalles de lo que está pasando en las diversas situaciones de enseñanza; así, la metodología propia del momento actual de las didácticas es cualitativa (Rist, 1982). Sin embargo, se elaboran también modelos teóricos (MD) que dan cuenta de situaciones idealizadas y que permiten ir consolidando un núcleo teórico propio y afinando el significado de los conceptos que siempre han servido para caracterizar la enseñanza: evaluación, profesor, alumno, actitud, valor, estrategia de enseñanza y rendimiento escolar.

Los didactólogos planifican la enseñanza de las ciencias a grupos o individuos de características diferentes e investigan los problemas que se derivan de ello. Disponen de una perspectiva de análisis y de una metodología de investigación, y ello es ya mucho para vertebrar una formación profesionalizadora. Los profesores e incluso los divulgadores de las ciencias deben ser formados desde este conocimiento, es decir, deben ser formados por didactólogos. Por ello, el campo profesional propio de la didactología es la formación del profesorado y, en general, de todos aquellos que se ocupan de dar a conocer las ciencias a la población no especialista. (Azcárate et al., 1993) (Furió y Gil, 1989). Se fomenta así una actitud atenta e investigadora en el aula, y ofrece recursos para ella, enriqueciendo así la investigación en didactología.

A partir de esta última reflexión podemos diferenciar entre profesor de ciencias y didactólogo. El interés por los problemas que aparecen al enseñar 
ciencias puede llevar a un profesor a profundizar en sus conocimientos, a interesarse por lo que pasa en otras situaciones similares o diferentes a la suya y a investigar para comprender los mecanismos del proceso de enseñanza. Todo ello le capacitará progresivamente para formar a otros profesores, para ayudarles en su tarea y con ello, realizar una actividad como didactólogo.

Un aspecto que parece digno de destacarse es la función importantísima que tiene la enseñanza de una ciencia en su estructuración teórica y en la ordenación, selección y formación de sus conceptos clave. La labor de los profesores de ciencias nunca fue pasiva sino que influyó fuertemente en la propia evolución del conocimiento científico; en palabras de López Rupérez (1990), la ciencia "se vierte en su didáctica". Por eso la reflexión teórica propia de la didactología coincide en parte con la que investiga el contexto de desarrollo de las teorías científicas, que tiene una relación estrecha con la enseñanza de las ciencias.

\section{Bibliografía}

Aliberas, J., GutiérRez, R., Izquierdo, M. (1990): «Modelos de aprendizaje en la didáctica de las ciencias". Investigación en la Escuela, 9, 17-24.

ANDERSON, (1977): «The motion of schemata and the educational enterprise: general discussion of the conferencem. In R.C. Anderson, Shapiro, Mortagne (Eds.). Schooling and the Adquisition of knowledge. Hillsdale New York, Elbrain

AzCarate, C., Espinet, M., IzQuierdo, M., SANMARTI, N. (1993): «La didáctica de las ciencias experimentales y la didáctica de las matemáticas, unas disciplinas emergentes". Comunicación presentada en el Simposium: Las Didácticas Especificas en la Formación del Profesorado. Santiago de Compostela.

BENEDITO, V. (1987): Introducción a la Didáctica. Fundamentación teórica y diseño curricular. Barcelona: Barcanova.

BLACK, P., LuCAS, A. ed, (1993): Chidren's Informal Ideas in Science. London: Routledge.

BROUSSEAU, G. (1984): «Le role central du contract didactique dans l'analyse et la construction des situations d'enseignement et d'apprentissage des mathématiques», $3 r$ Ecole d'été de didactique des mathématiques, Olivet.

CAÑAL, P. (1987): «Un enfoque curricular basado en la investigación». Investigación en la escuela, 1, 43-50. 
Coll, C. (1986): Marc Curricular per a l'Ensenyament Obligatori. Departament d'Ensenyament, Generalitat de Catalunya.

DRIVER, R. (1986): «Psicologia cognoscitiva y esquemas conceptuales de los alumnos». Enseñanza de las Ciencias, 4 (1), 3-15.

ECHEVARRIA, J. (1995): Filosofia de la Ciencia. Madrid: Akal.

ESTANY, A. (1993): Introducción a la Filosofia de la Ciencias. Crítica: Barcelona.

FENSHAM, P. (1988): Development and dilemmas in science education. Salisbury: The Falmer Press.

FURIO, C., GIL, D. (1989): “La didáctica de las ciencias en la formación inicial del profesorado: una orientación y un programa teoricamente fundamentados». Enseñanza de las ciencias, 7 (3), 257-265.

GIERE, R. (1988): Explaining science. A cognitive approach. Chicago: University of Chicago Press.

Giordan, A. (1983): L'élève ou/ et les connaissances scientifiques. Berne: Peter Lang.

Gutiérrez, R. (1985): "La investigación didáctica en el área de Ciencias: ¿Nueva crísis de paradigmas?" Comunicación presentada en el ler. Congreso Internacional sobre investigación en la Didáctica de las Ciencias y de las Matemáticas. Bellaterra.

HABERMAS, J. (1971): "Toward a theory of communicative competence. En: Hans Peter Dreitzel (Ed) Recent Sociology, n. ${ }^{\circ}$ 2" New York: Mac Millan

Hodson, D. (1985): "Phylosophy of science, science an science education". Studies in Science Education, 12, 25-57.

Hodson, D. (1987): "Social control as a factor in science comunical change». International Journal Science Education, 9 (5), 529-550.

HosT, V. (1982): «El lugar de los aprendizajes espontáneos en la formación científicai. Infancia y Aprendizaje, 19-20, págs. 3-20.

IZQUIERDO, M. (1990): «Bases epistemològiques de l'ensenyament de les ciències». Edhucar, 17, 69-90.

IZQUIERDO, M. (1994a): "Cognitive Models of Science and Science Education". Sumer School: Research in Science Education. Thessaloniki.

IZQUIERDO, M. (1994b): "Las ciencias de la naturaleza en la ESO ¿̇un área común o disciplinas distintas?». Infancia y Aprendizaje, 65, 31-34.

Johnson-Laird, P. N. (1988): The Computer and the Mind. Fontane: Fontane Press. JORBA, J., SANMARTI, N. Enseñar, aprender y evaluar: un proceso de regulación continua. Madrid: MEC. (En prensa). 
JoshuA, S., Dupin, J. J. (1993): Introduction à la didactique des sciences et des matheématiques. Paris: PUF.

KRUGLY-SMOLSKA, E. (1995): "Cultural influences in science education". International Journal Science Education, 17(1), 45-58.

LOPEZ RUPÉREZ, F. (1990): “Epistemología y Didáctica de las Ciencias. Un análisis de segundo orden". Enseñanza de las Ciencias, 8(1), 65-74.

MINSKY, M. (1988): The society of mind. Madrid: Picador.

NeWTON-SMITH, W. H. (1987): La racionalidad de la ciencia. Barcelona: Paidós.

NiINILOUTO, I. (1993): "The aim of applied research». Erkentniss, 38, 1-21.

NovaK, J. D. (1982): Teoria y Práctica de la Educación. Madrid: Alianza.

OGBORN, J. (1985): “Understanding student's understandings: An example from dynamicsm. European Journal Science Education, 7(2), 141-150.

Pozo, J. I. (1989): Teorias cognitivas del Aprendizaje. Madrid: Morata.

RIST, R. C. (1982): "On the application of ethnografic inquiry to education: procedures and possibilities». Journal of Research in Science Teaching, 19(6), 439-450.

Rumelhardt, D. E., Norman, D. A. (1978): "Accretion, tunning and reestructuring». En: Semantic factors in cognition. Hillsdall N.Y.: Erlbaum.

SCHANK, R. C., ABeLSON, R. P. (1977): Scripts, plans, goals and understanding. An inquiry into human kwowledge structures. New Jersey: LEA.

STEWART, J. (1985): "Cognitive Science and Science Education". European Journal Science Education, 7(1), 1-17.

WRIGHT, G. H. von, (1979): Norma y acción. Madrid: Textos.

WRIGHT, G. H. von, (1983): Practical reason. Oxford: Basil Blackwell.

YaGeR, R. E. Bybee, B., Gallager, J. J., ReNneR, J. W., (1982): "An Analysis of the Current Crisis in the Discipline of Science Education". Journal of Research in Science Teaching, 19 (5), 377-395. 